\title{
Occupant behavior modelling to support the development of adaptive facade control strategies
}

\author{
Isabella Gaetani $^{1,2}$, Remco van Woensel ${ }^{1}$, Pieter-Jan Hoes ${ }^{1}$, Jan L.M. Hensen ${ }^{1}$ \\ ${ }^{1}$ Building Physics and Services, Eindhoven University of Technology, P.O. Box 513, 5600 MB \\ Eindhoven, The Netherlands \\ ${ }^{2}$ Arup, 13 Fitzroy Street, London, W1T 4BQ, United Kingdom
}

\begin{abstract}
This study investigates the appropriate model complexity for occupancy models when assessing the performance of presence-dependent control strategies for adaptive solar shading systems.

BPS tools are useful during the $R \& D$ process of innovative, solar shading control strategies. However, inappropriate consideration of key aspects of occupant behavior (OB) in the BPS tools could lead to suboptimal design decisions. Therefore, a previously developed methodology was applied to select fit-for-purpose OB models for different solar shading control strategies.

The results show that a refined occupancy model is needed when evaluating the energy performance of the best-performing strategy, while it would be redundant in all other cases. Nevertheless, considering a variety of scenarios as opposed to a single occupancy schedule is essential to informed decision-making.
\end{abstract}

\section{Introduction}

Adaptive façade elements traditionally adapt to their surrounding environment. However, to exploit fully the potential of adaptive architecture, it is advisable that these adaptive elements also react to occupancy (Cole and Brown 2009). In this way, it would be possible to optimize the building operation towards comfort when people are present and towards energy use minimization when people are absent.

BPS tools have a strong potential to be used to support the development of innovative, adaptive solar shading systems (Loonen et al. 2017). The advantages of using such tools already in the R\&D phase are: i) virtual rapid prototyping to evaluate different future-oriented systems/materials and identifying promising alternatives for further refinement and product development; ii) exploration of high-potential control strategies that maximize the performance of adaptive building envelopes during operation; iii) virtual testing of the robustness of adaptive façade systems to changing boundary conditions (Loonen et al. 2017).

Meanwhile, the assumptions made in the BPS model are determinants of the outcome of the simulation (Hopfe and Hensen 2011; Rezaee et al. 2015). For this reason, it is essential that the assumptions are as conscious and informed as possible. For example, if a simulation user is interested in supporting the R\&D of adaptive façade elements that also react to occupancy, an appropriate representation of occupancy is critical. While some existing studies consider the human factor in adaptive facades simulation (e.g., (Bakker et al. 2014)), no currently available method supports the choice of OB models during the R\&D of adaptive façade elements.

This study seeks to fill this gap by applying a previously developed method, the fit-for-purpose occupant behavior modeling (FFP-OBm) method (Gaetani, Hoes, and Hensen 2017), to adaptive façade elements currently under development by industrial partners.

To achieve this goal, the FFP-OBm is applied with a double aim: i) to assess which control strategy delivers the best building performance; ii) to evaluate the performance of the best shading strategy. This operation, if successful, should lead to an informed choice of occupancy models during the R\&D phase of adaptive façade elements.

\section{Methodology}

The case study

Shading strategies characteristics

Six control strategies, corresponding to state-of-the-art controls or controls that are under development by industrial partners, are evaluated. The strategies are described below.

- Strategy 1 two blind positions available (fully up or fully down); irradiance sensor on the roof; lowering threshold $300 \mathrm{~W} / \mathrm{m}^{2}$;

- $\quad$ Strategy 2 two blind positions available (fully up or fully down); irradiance sensor on the façade; lowering threshold $200 \mathrm{~W} / \mathrm{m}^{2}$;

- Strategy 3 two blind positions available (fully up or lowered to eye height); irradiance sensor on the façade; lowering threshold $200 \mathrm{~W} / \mathrm{m}^{2}$;

- Strategy 4 twenty blind positions available (full height discretized in 20 identical parts); sun-tracking feature to avoid direct sunlight;

- Strategy 5 same as Strategy 4 but includes an energy mode (see Table 1) when occupants are absent;

Table 1: Shades control in energy mode

\begin{tabular}{|c|c|c|}
\hline & $\begin{array}{c}\text { Heat gain through } \\
\text { façade }\end{array}$ & $\begin{array}{c}\text { Heat loss through } \\
\text { façade }\end{array}$ \\
\hline $\begin{array}{l}\text { Heating } \\
\text { demand }\end{array}$ & Shades Up & Shades Down \\
\hline $\begin{array}{l}\text { Cooling } \\
\text { demand }\end{array}$ & Shades Down & Shades Up \\
\hline
\end{tabular}


- $\quad$ Strategy 6 same as Strategy 5 but includes overcast sky feature, whereby if the room is occupied and the vertical solar irradiance on the façade is below 120 $\mathrm{W} / \mathrm{m}^{2}$, the blinds are fully raised.

A graphical representation of the six shading strategies is given in Fig. 1. Two additional strategies, namely "fully up" and "fully down" were added to the analysis for the sake of benchmarking.
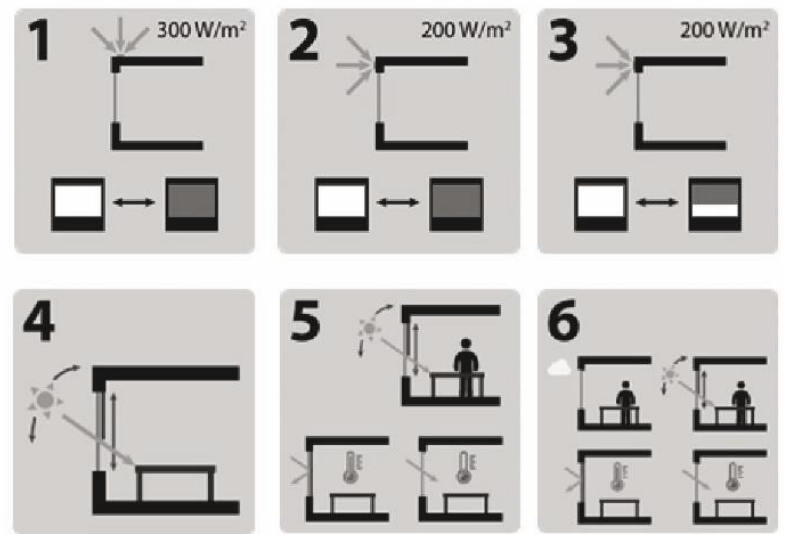

Figure 1: Graphical representation of the six considered shading strategies

The solar shading control strategies were modeled by means of the Energy Management System (EMS) feature of EnergyPlus. While the authors are aware that other available software may be more specifically tailored for daylight analysis (e.g., Radiance), this choice was made to simplify the case study. All strategies were applied to an automated solar shading system developed by industrial partners. The system is an internal roller blind with a metalized fabric, whose thermal properties are reported in Table 2.

\section{Table 2: Thermal properties of the roller blind}

\begin{tabular}{|c|c|c|c|c|c|}
\hline $\begin{array}{c}\text { Thickness } \\
{[\mathbf{m}]}\end{array}$ & $\begin{array}{c}\text { Thermal } \\
\text { conductivity } \\
{[\mathbf{W} / \mathbf{m K}]}\end{array}$ & $\begin{array}{c}\text { Sol. } \\
\text { Tran } \\
\text { s. }\end{array}$ & $\begin{array}{c}\text { Sol. } \\
\text { Refl. }\end{array}$ & $\begin{array}{c}\text { Vis. } \\
\text { Trans. }\end{array}$ & $\begin{array}{c}\text { Vis. } \\
\text { Refl. }\end{array}$ \\
\hline 0.0004 & 0.2 & 0.078 & 0.648 & 0.077 & 0.632 \\
\hline
\end{tabular}

\section{Performance Indicators}

A variety of performance indicators (PIs) are considered, related to both the energy and the comfort performance of the shading system. The selected PIs are:

- Annual primary energy demand; considered as a sum of heating, cooling and lighting energy demand $\left[\mathrm{kWh} / \mathrm{m}^{2}\right]$, taking into account the conversion factors reported in Appendix A;

- Daylight Autonomy (DA); calculated in two reference points (see Appendix B) and expressed in occupied time fraction when the indoor illuminance is > 500 lux (Walkenhorst et al. 2002);
- Daylight Glare Index (DGI); calculated according to (Hopkinson 1972) and expressed in occupied time fraction when DGI > 31, corresponding to intolerable glare. The DGI is calculated for three viewpoints, namely "Facing Window", "Facing Corner" and "Facing Wall" (see Appendix B).

\section{Space characteristics}

A two-occupant cubicle office located in Amsterdam, The Netherlands, and having dimensions of $4.5 \times 6 \times 3 \mathrm{~m}^{3}$ (see Fig. 2) was considered to evaluate the performance of the shading strategies within a space application. The space is a modified version of the reference office proposed by Task 56 of the Solar Heating and Cooling Program of the International Energy Agency (Antoni et al. 2018). This space is regularly used for testing purposes, but it is clearly a simplification. Other spaces (e.g. classrooms, open-plan studios) with different occupancy should be investigated, as they will likely lead to different results.

The cubicle is assumed to be surrounded by similar offices in thermal equilibrium with it, hence all surfaces but the wall facing outside are modeled as adiabatic. A large non-operable window is located on the external wall. The thermo-physical properties of the space follow the references of Task 56 (Antoni et al. 2018).

The space is modeled in EnergyPlus V8.7.

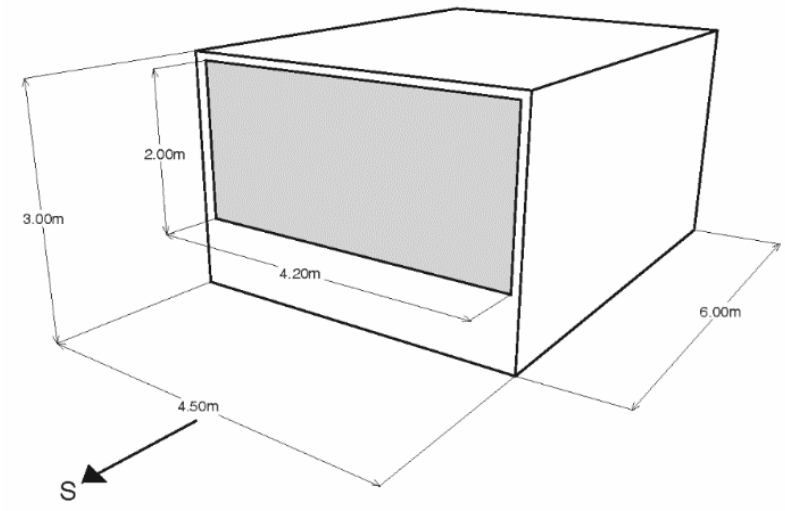

Figure 2: The cubicle office

The light power density is set to $10.9 \mathrm{~W} / \mathrm{m}^{2}$. The heat emitted by lights is assumed to be $13 \%$ radiant. The lights are dimmed according to illuminance levels and occupancy (all lights are off if nobody is present). The power density of appliances is set to $7 \mathrm{~W} / \mathrm{m}^{2}$ with $30 \%$ radiant heat. The reference schedule for occupancy is given in Fig. 3, while the reference schedule for equipment use is ASHRAE standard (ASHRAE 2013).

\section{The FFP-OBm method}

As mentioned, the FFP-OBm method (Gaetani, Hoes, and Hensen 2016) is applied to determine the appropriate model for occupancy. In the method, models are grouped according to their complexity, from simple schedules, deterministic and non-probabilistic models (complexity 0 ) to stochastic models (1), and agent-based models (2). 
In this study, we assume that higher complexity models yield results that are more realistic. We understand that this assumption may not always be true, since the commonly used OB models often have not undergone a rigorous validation process (Mahdavi and Tahmasebi 2016) and the input required by complex models may not always be readily available (Gaetani, Hoes, and Hensen 2016). Nonetheless, we expect that in the future all models connected to the FFP-OBm method will be reliable within their application range.

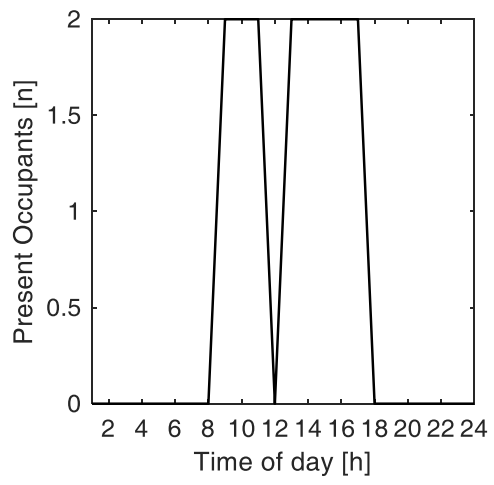

Figure 3: Reference schedule for occupancy

Fig. 4 is an overview of the methods employed by the FFP-OBm framework. Two methods of the framework are not applicable for this case study: the Impact Indices method, developed for other PIs than the ones considered here, and the Mann-Whitney $U$ test, which is useful when multiple aspects of $\mathrm{OB}$ are considered.

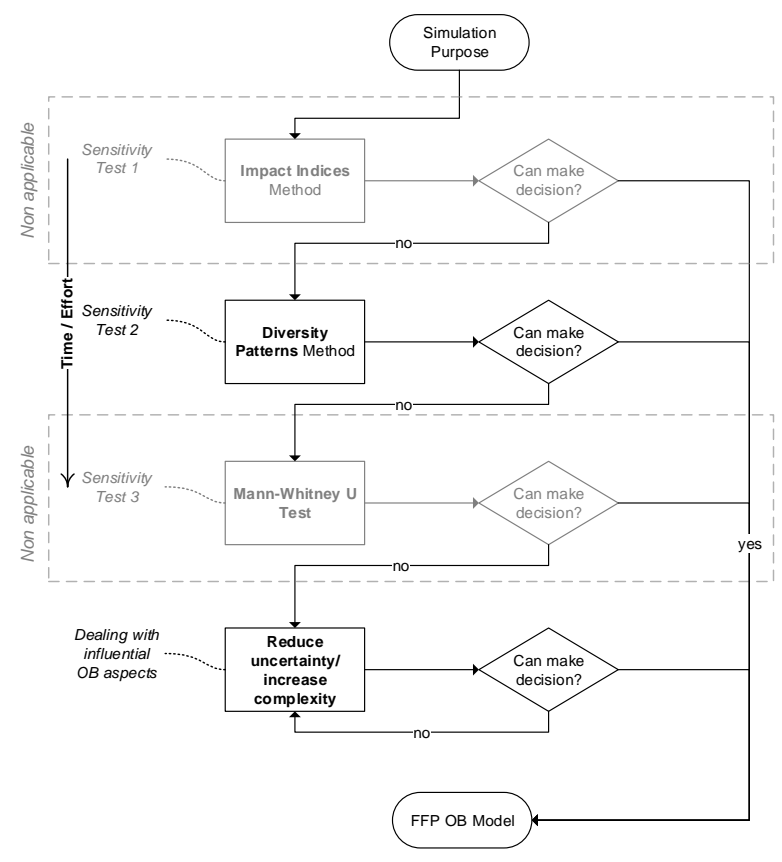

Figure 4: Overview of the methods employed by the FFP-OBm framework

Hence, the methods that are applied to this case study are the Diversity Patterns method (Sensitivity test 2 ) and the
Reduce uncertainty/increase complexity method. The Diversity Patterns method consists in applying high-low, extreme variations of the $\mathrm{OB}$ aspect. The Reduce uncertainty/increase complexity method involves a finetuning of the representation of the OB aspect, possibly reducing the uncertainty connected to such aspect(s), or increasing the complexity of its connected model. The latter method occurs if one or more OB aspects are identified as influential for the purpose of the simulation.

\section{The occupancy models}

Two levels of model complexity are considered: complexity " 0 ", or simple schedules, and complexity " 1 ", a stochastic occupancy model by Page et al. (2008). The application of diversity patterns consists de facto in adding diversity to simple schedules.

Page et al.'s discrete-time, non-homogeneous Markov model allows generating the annual presence time-series at 15 min timesteps. This model requires two parameters: the weekly occupancy schedule and the parameter of mobility. If the user is not aware of a building-specific occupancy schedule, an option is to input the ASHRAE schedule (ASHRAE 2013) as weekly occupancy schedule. However, the ASHRAE schedule potentially takes over-hours into account, as the presence probability after $7 \mathrm{pm} \neq 0$ (see Fig. 5 left). Moreover, it is worth mentioning that the implementation of probabilistic OB models in deterministic BPS tools often occurs by comparing the probability computed by the model with a random number at each simulation timestep. In the case of occupancy, if the model probability is higher than the random number, occupants turn out to be present. This workaround may result in non-significant patterns if a schedule is used to derive transitions (as is the case here, where the ASHRAE schedule is used as input), because occupancy is questioned every $15 \mathrm{~min}$. Therefore, two options are explored hereafter: the standard ASHRAE schedule (Fig. 5 left), and a modified schedule (Fig. 5 right) whereby people are absent before 8 am and after 7 pm. The assumption of not considering over-hours is made also when developing the diversity patterns (see Fig. 9).
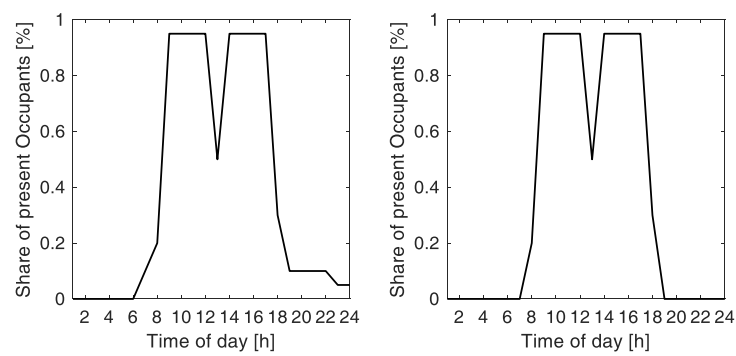

Figure 5: Standard ASHRAE occupancy fraction (left), and modified as absence before 8 am and after $7 \mathrm{pm}$ (right)

The value for the parameter of mobility is taken from (Gunay, O’Brien, and Beausoleil-Morrison 2016). 


\section{Results and discussion}

\section{Preliminary results}

Due to the complexity of the strategies and the necessity to use advanced features of EnergyPlus, the results of a preliminary evaluation run are reported below. An analysis of such preliminary results serves to check that all strategies are behaving as expected and to allow troubleshooting when necessary.

\section{Annual primary energy demand}

Fig. 6 shows the strategies' energy performance when standard assumptions about occupancy are made. The heating energy demand is hardly affected by the strategy, with a discrepancy of $2.6 \mathrm{kWh} / \mathrm{m}^{2} \mathrm{y}$ between the highest (S2) and lowest (S1) demand among the strategies. This result is in line with the fact that heating energy demand is the lowest contributor to primary energy demand. More pronounced differences can be seen in the cooling demand, where the maximum discrepancy among strategies rises to $10.1 \mathrm{kWh} / \mathrm{m}^{2} \mathrm{y}$ ( $\mathrm{S} 6$ has the lowest demand, while $\mathrm{S} 4$ has the highest). The biggest difference among strategies is found in the lighting energy demand: $11.5 \mathrm{kWh} / \mathrm{m}^{2} \mathrm{y}$ (demand of $\mathrm{S} 4,5-\mathrm{S} 3$ ). It is worth noting the effect of implementing "Energy Mode", which reduces the cooling energy demand from 53.3 to 43.8 $\mathrm{kWh} / \mathrm{m}^{2} \mathrm{y}$ in S4 and S5, respectively. The "Overcast Sky" feature results in a reduction of lighting energy demand of $10.7 \mathrm{kWh} / \mathrm{m}^{2} \mathrm{y}$ from S5 to S6. Overall, the strategies work as intended. The best-performing strategy in terms of energy is S6, while the worst performing strategy is S4. S4 demands circa $27 \%$ more primary energy than S6.

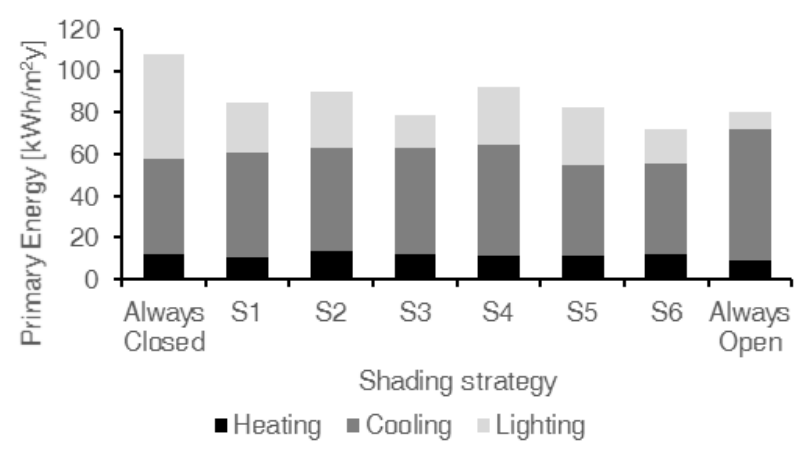

Figure 6: Energy performance of the six strategies with standard occupancy schedules

\section{Daylight autonomy}

Fig. 7 shows the strategies' daylight autonomy performance when standard assumptions about occupancy are made. S4 and S5 deliver the same performance, as expected, because the difference between the two strategies concern only the unoccupied hours.

The benefit of the "Overcast Sky" feature is evident when comparing the performance of S6 with S4 and S5. Out of all strategies, S3 guarantees the best DA $(87 \%$ of occupied hours $>500$ lux), followed by S6 (81\% of occupied hours > 500 lux), while S4 and S5 only guarantee an illuminance of 500 lux in $63 \%$ of the occupied hours.

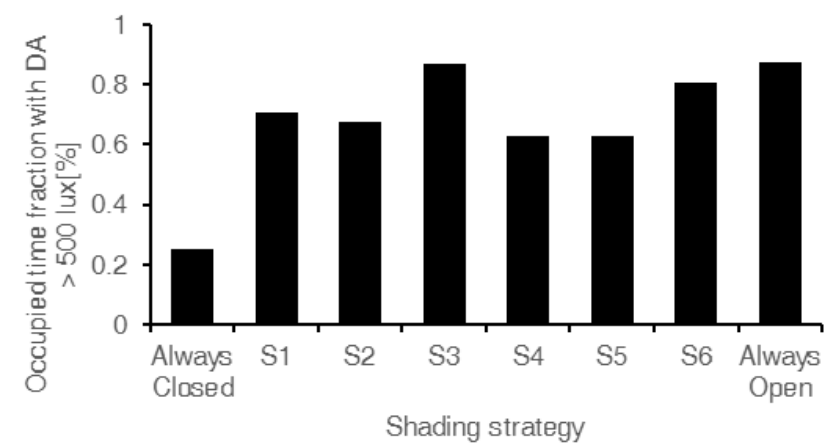

Figure 7: Daylight autonomy performance of the six strategies with standard occupancy schedules

\section{Daylight Glare Index}

The glare performance of the various strategies with standard assumptions about occupancy are shown in Fig. 8 for three different viewpoints.

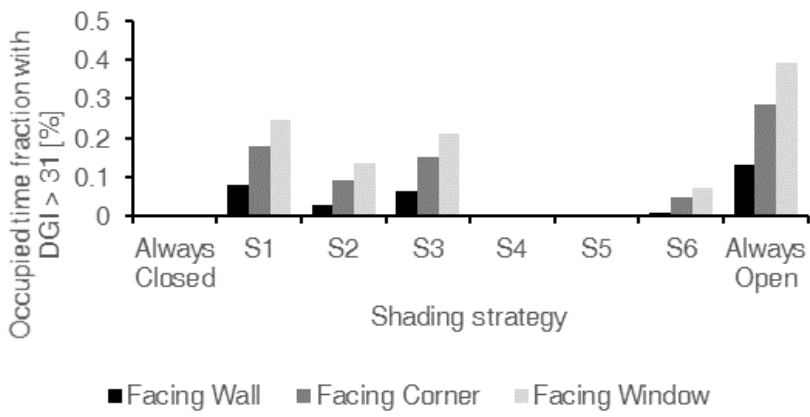

Figure 8: DGI performance of the six strategies with standard occupancy schedules

As expected, the viewpoint "Facing Window" is characterized by the highest glare, followed by "Facing Corner" and, lastly, "Facing Wall". Whether or not the amount of glare indicated by the software is acceptable will therefore depend on the envisioned layout of the office. The benchmark option "Always Open" delivers the worst performance, leading to about $40 \%$ of occupied hours with intolerable glare when facing the window. This value decreases to ca. $13 \%$ when the occupant faces the wall instead. According to this PI, the options "Always Closed", S4 and S5 are completely glare-free. This result is expected, as the mentioned strategies are purposively developed to avoid glare. In contrast, S6 is characterized by a low amount of intolerable glare, indicating that the sky illuminance may also be enough to cause glare when the sky is overcast. Overall, S1 leads to the worst performance with about $25 \%$ hours of intolerable glare while facing the window, while S4 and S5 are the best performing.

In the following sections, it is investigated whether these results are presence-sensitive, and if higher modeling complexity is needed to reach reliable conclusions.

\section{Diversity Patterns Method}

The fit-for-purpose complexity of occupancy modeling is at the heart of the investigation. Therefore, diversity patterns are defined only for occupancy. The operation of the shading devices is automated by means of the 
strategies, hence it does not require the formulation of diversity patterns. Likewise, the lighting system is supposed to be automatic, and the HVAC system is centrally controlled. The use of appliances is, together with occupancy, the only type of behavior that is left to the occupants' discretion in this case study. However, we decided to focus on occupancy modeling to narrow down the scope.

The developed diversity patterns are shown in Fig. 9. In particular, they correspond to a rather rigid work environment where people have a maximum deviation of $\pm 2 \mathrm{~h}$ from the reference schedule. In order to evaluate the robustness of the diversity patterns, two possible combinations, named Option 1 and Option 2 are developed. In Option 1, the occupants arrive one hour sooner/later than the reference and leave one hour sooner/later. Alternatively, in Option 2, the highly present workers work through lunchbreak and stay one hour longer in the evening, while the workers spending least time in the office take a three-hour lunchbreak. These two options are developed to assess the impact of considering hours characterized by different illuminance levels.

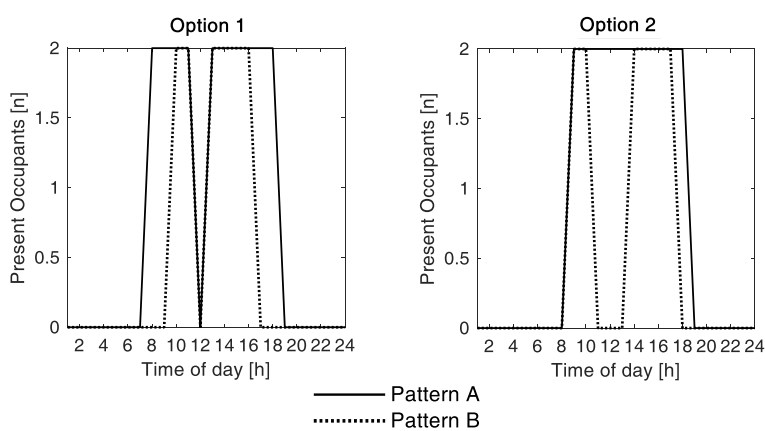

Figure 9: Two options of Diversity Patterns for occupancy: Option 1 (left) and Option 2 (right)

Once the Diversity Patterns are developed, the simulations are run and the results are processed.

\section{Annual primary energy demand}

Fig. 10 shows the effect of applying the diversity patterns on the annual primary energy demand. The results show how the robustness to occupancy varies significantly across strategies. In particular, the benchmarking option "Always Closed" shows the highest sensitivity to occupancy, while the option "Always Open" shows the least sensitivity. This effect is largely imputable to the energy demand for lighting, which in the "Always Closed" case depends solely on occupancy, while it is significantly influenced by available daylight in the "Always Open" case. The increased effect of occupancy on S5 and S6 (which included the "Energy Mode" feature when occupants are absent) is somewhat visible. Fig. 9 also shows the importance of the defined diversity patterns. A change of occupancy during the first and last hours has a stronger effect than during the mid-hours of the day. Generally, the effect of occupancy on the strategies' energy performance is visible in all cases. Whether such an effect is acceptable or a higher modeling complexity is required will be up to the decision-maker (see following section).
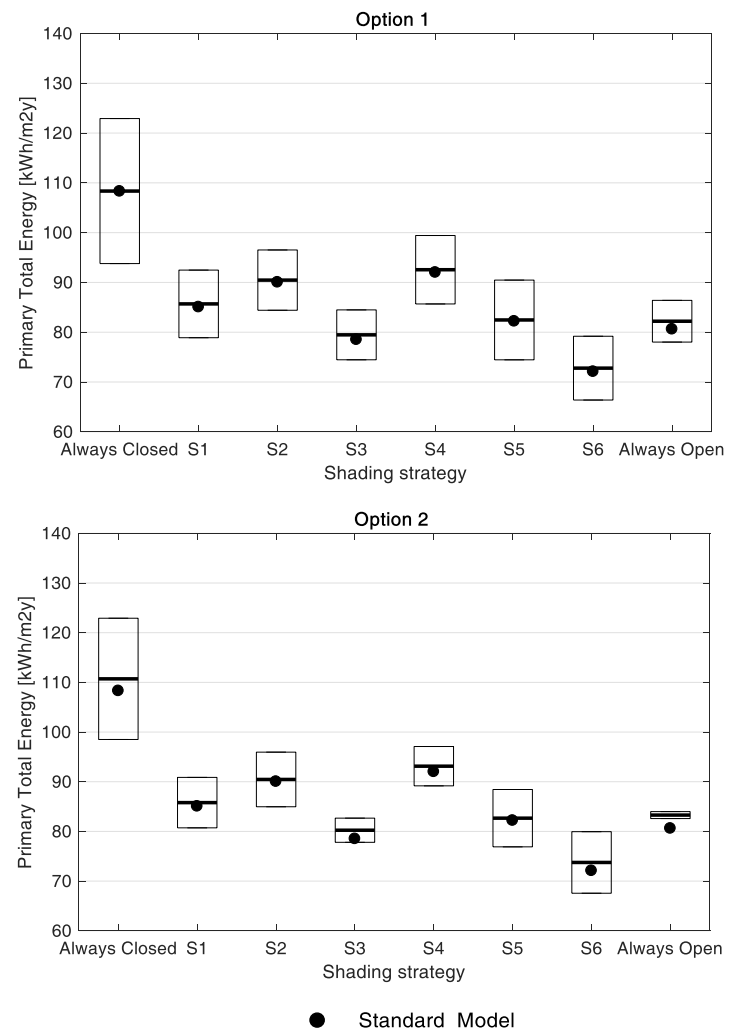

Figure 10: Effect of Diversity Patterns on the annual primary energy demand. Option 1 (top) and Option 2 (bottom)

\section{Daylight autonomy}

Fig. 11 shows the effect of applying the diversity patterns on the daylight autonomy. The results show a similar robustness to occupancy across strategies. Also in this case a change of occupancy during the first and last hours has a stronger effect than during the mid-hours of the day. In fact, the PI is very robust to the mid-hours Diversity Patterns.

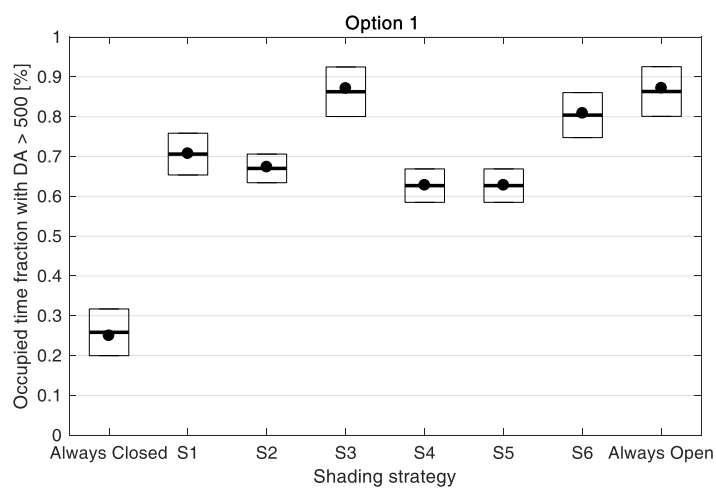




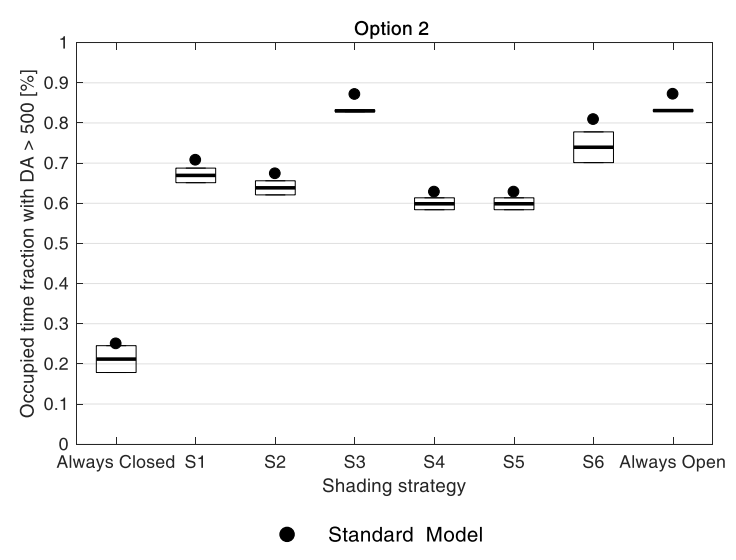

Figure 11: Effect of Diversity Patterns on the daylight autonomy. Option 1 (top) and Option 2 (bottom)

This effect is explained given the southern orientation of the large window: the differences in natural daylight, especially when a threshold of 500 lux is considered, are expected to be stronger during the first and last hours of the day, rather than during the central hours. The effect of occupancy on decision-making is considered hereafter.

\section{Daylight Glare Index}

Fig. 12 shows the effect of applying the Diversity Patterns on the glare performance of the six strategies.
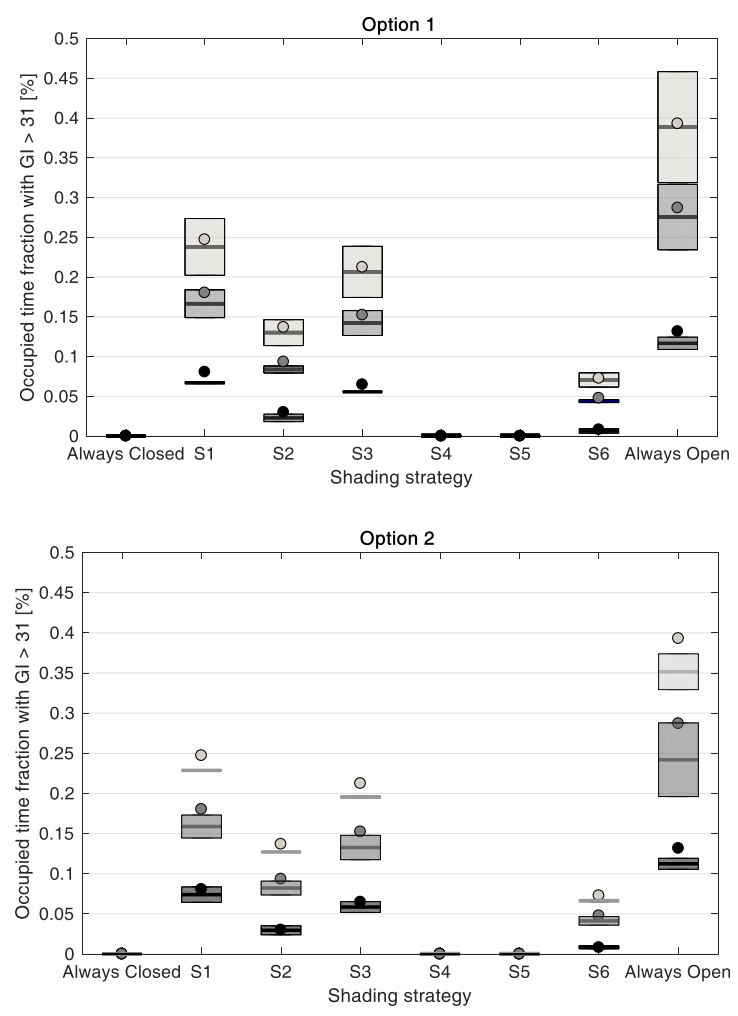

Standard Model - Facing Window

- Standard Model - Facing Wall

- Standard Model - Facing Corner

Figure 12: Effect of Diversity Patterns on the glare performance. Option 1 (top) and Option 2 (bottom)
The results show a diverse robustness to people presence across strategies. In particular, the most sensitive options are the benchmark "Always Open" and S1, S2, S3. The option "Always Closed" and the solutions with sun tracking (S4, S5 and S6) guarantee the lowest amount of occupied hours characterized by intolerable glare and are robust to people presence.

\section{Decision-making}

Assessing which control strategy delivers the best performance

Despite the different sensitivities across strategies to occupancy, especially pronounced for annual primary energy demand and glare performance, it is possible to take a decision in all cases. In particular, the ranking of strategies from best to worst performance (see Fig. 13) is unaffected by the application of Diversity Patterns. For this reason, it is suggested not to increase the level of complexity for occupancy modeling if the aim is to assess which control strategy delivers the best performance. Out of all strategies, the only one that remains in the top 4 (considered as the "good performance zone") for all PIs is S6 (Fig. 13). However, it will be up to the company to prioritize PIs and/or ease and cost of development. These priorities will inform the choice of which strategy to favor.

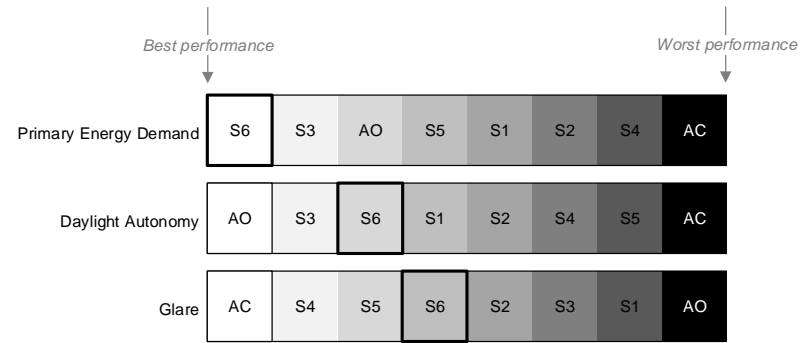

Figure 13: Ranking of strategies performance from best to worst. $A O$ and AC indicate "Always Open" and "Always Closed”, respectively

Evaluating the performance of the best control strategy The need for refining the model complexity of occupancy depends on the desired accuracy of the results.

For example, we may conclude that the spread in annual primary energy demand obtained by means of the Diversity Patterns is too high. The occupancy Pattern A (Option 1) led to a primary energy demand of 79.2 $\mathrm{kWh} / \mathrm{m}^{2} \mathrm{y}$, while Pattern B indicated that the space would need only $66.4 \mathrm{kWh} / \mathrm{m}^{2} \mathrm{y}$ to operate during the same period. Assuming that Pattern A and Pattern B are extreme conditions, we may attempt reducing the variation of these results by refining the occupancy model.

Instead, in the case of comfort PIs, building codes typically exist that define the minimum acceptable percentage of occupied hours within a given threshold. (CIBSE 1994), for example, sets the minimum percentage of occupied hours with an illuminance level above 500 lux at $75 \%$. This kind of compliance-driven calculations would normally come with a standard occupancy 
schedule. Nevertheless, it is interesting to determine whether such limits would be achieved also in more extreme presence scenarios, as hypothesized by the Diversity Patterns.

An analysis of the response of the strategies to presence illustrates how only two strategies comply with this limit in all cases: S3 and S6. For these two strategies, it is hence not required to increase the presence model complexity, as they always fall within the required limits.

Likewise, one may seek to limit the number of occupied hours with intolerable glare at $10 \%$. Of all investigated strategies, only S4, S5 and S6 fall within this threshold while facing the window. Therefore, for these three strategies it is not required to increase the complexity of the occupancy model.

To conclude, a refinement of the occupancy model (in this study coinciding with the application of higher model complexity) is necessary only when evaluating the energy performance of S6.

\section{Higher model complexity}

As mentioned, the selected higher complexity model for occupancy is the stochastic model by Page et al.

Fig. 14 shows the results obtained by running the Page model 50 times according to the occupancy probability schedules presented in Fig. 5. The reasoning behind the value of 50 can be found in (Feng, Yan, and Wang 2016).

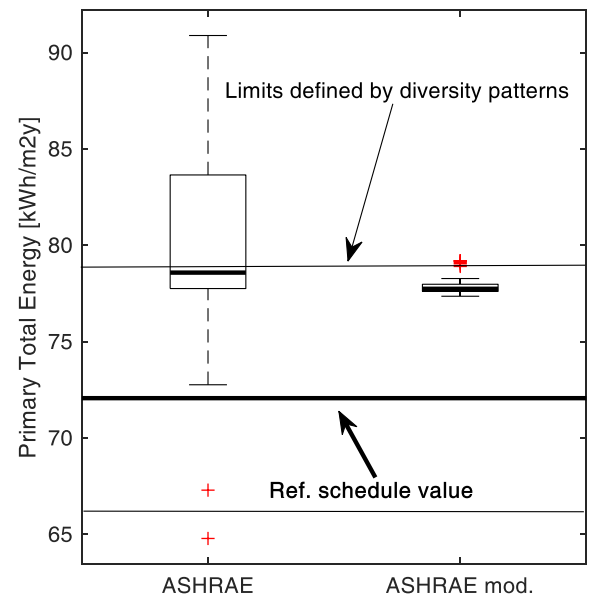

\begin{abstract}
Figure 14: Energy performance of S6 when implementing Page et al. 's model to the ASHRAE schedule and the modified AHSRAE schedule
\end{abstract}

The median values of energy demand according to the Page model are closer to the upper limit of the diversity patterns (see Fig. 10). This result is in line with expectations, as the main share of energy demand is imputable to lighting energy, which is occupantdependent. In both cases illustrated in Fig. 5, it rarely occurs that no occupant is present between 9 am and $6 \mathrm{pm}$. It is worth noting the differences in results according to the assumed probability distribution function. In particular, assuming the standard ASHRAE schedule as a probability distribution function for occupancy leads to higher uncertainty than the defined Diversity Patterns. This result is mainly caused by the after-hours. Instead, the results obtained with the modified ASHRAE schedule are quite robust to occupancy, and are close to the upper limit indicated by the diversity patterns, as expected. This result can be explained as the major differences across simulation runs concern whether one or two occupants are present, and not whether the space is occupied or not.

To conclude, implementing a higher model complexity for presence in this case revealed that the expected energy performance of S6 is closer to the upper limit of the diversity patterns, rather than to the median or lower limit. The decision-maker should then assess whether the actual occupancy probability distribution function is closer to the ASHRAE schedule or to the modified ASHRAE schedule. By doing so, it is possible to decide if S6 leads to a satisfactory performance or not.

\section{Conclusions}

This study applies the FFP-OBm method, a method to support OB model selection, to the R\&D of adaptive, presence-dependent, innovative shading elements. Six different shading control strategies are evaluated in terms of energy and visual comfort performance. The FFP presence model is identified for each strategy and each PI.

The results show that most PIs are sensitive to the assumptions made regarding occupancy. The only strategy/PI that are robust to occupancy assumptions are the glare results for S4 and S5. Both these strategies were developed to avoid glare, and indeed, they deliver the required performance independently of the assumptions made for occupancy. Despite the different response of various shading strategies to occupancy, a refinement of the presence model was not necessary to rank their mutual performance for all investigated PIs. In contrast, it may be needed when calculating the actual performance of a single strategy. In this case study, the stochastic occupancy model from Page et al. (2008) helps establishing that the actual performance is likely be closer to the upper limit identified by the diversity patterns. This result is achieved despite neglecting over-hours. Further, this case study highlights the importance of carefully considering the assumptions made while modeling occupancy. Two different pairs of diversity patterns have a different impact on the strategies/PIs. Moreover, two different schedules input to the Page et al. model yield drastically different results.

In conclusion, this case study identifies a potential for the FFP-OBm to be used during the R\&D process of adaptive façade elements. This study combines $\mathrm{OB}$ modeling and adaptive façade elements research. It is a step towards the development of robust methods to assess the actual performance of adaptive elements, which is influenced by uncertain variables such as presence. Secondly, it contributes to the lively discussion in the BPS community regarding fit-for-purpose OB modeling.

\section{Acknowledgements}

The support of PIT/VABI, the SPARK consortium and TKI EnerGO TRECO-office is greatly appreciated. 


\section{References}

Antoni, D M, P Bonato, F Ochs, M Magni, De Vries S, R C G M Loonen, and R Fedrizzi. 2018. "Definition of a Reference Office Building for Simulation Based Evaluation of Solar Envelope Systems." In EuroSun 2018 - 12th International Conference on Solar Energy for Buildings and Industry. Rapperswil (Switzerland).

ASHRAE. 2013. ANSI/ASHRAE/IESNA Standard 90.12013: Energy Standard for Buildings except for LowRise Residential Buildings.

Bakker, L. G., E. C. M. Hoes-van Oeffelen, R. C. G. M. Loonen, and Jan L. M. Hensen. 2014. "User Satisfaction and Interaction with Automated Dynamic Facades: A Pilot Study." Building and Environment 78. Elsevier Ltd: 44-52. doi:10.1016/j.buildenv.2014.04.007.

CIBSE, CODE. 1994. "Code for Interior Lighting." London: The Chartered Institute of Building Services Engineers.

Cole, Raymond J., and Zosia Brown. 2009. "Reconciling Human and Automated Intelligence in the Provision of Occupant Comfort." Intelligent Buildings International 1 (1): 39-55. doi:10.3763/inbi.2009.0007.

Feng, Xiaohang, Da Yan, and Chuang Wang. 2016. “On the Simulation Repetition and Temporal Discretization of Stochastic Occupant Behaviour Models in Building Performance Simulation." Journal of Building Performance Simulation 0 (0). Taylor \& Francis: 113. doi:10.1080/19401493.2016.1236838.

Gaetani, Isabella, Pieter-Jan Hoes, and Jan L. M. Hensen. 2016. "Occupant Behavior in Building Energy Simulation: Towards a Fit-for-Purpose Modeling Strategy." Energy and Buildings 121. Elsevier B.V.: 188-204.

doi:http://dx.doi.org/10.1016/j.enbuild.2016.03.038

Gaetani, Isabella, Pieter-Jan Hoes, and Jan L. M. Hensen. 2017. "Introducing and Testing a Strategy for Fit-forPurpose Occupant Behavior Modeling in a Simulation-Aided Building Design Process.” In Building Simulation 2017, In Publication. San Francisco.

Gunay, H Burak, William O'Brien, and Ian BeausoleilMorrison. 2016. "Implementation and Comparison of Existing Occupant Behaviour Models in EnergyPlus." Journal of Building Performance Simulation 9 (6): 567-588. doi:10.1080/19401493.2015.1102969.

Hopfe, Christina J., and Jan L M Hensen. 2011. "Uncertainty Analysis in Building Performance Simulation for Design Support." Energy and Buildings 43 (10). Elsevier B.V.: 2798-2805. doi:10.1016/j.enbuild.2011.06.034.

Loonen, Roel C G M, Fabio Favoino, Jan L M Hensen, and Mauro Overend. 2017. "Review of Current Status, Requirements and Opportunities for Building
Performance Simulation of Adaptive Facades." Journal of Building Performance Simulation 10 (2): 205-223. doi:10.1080/19401493.2016.1152303.

Mahdavi, Ardeshir, and Farhang Tahmasebi. 2016. "On the Quality Evaluation of Behavioural Models for Building Performance Applications." Journal of Building Performance Simulation 0 (0). Taylor \& Francis: 1-11. doi:10.1080/19401493.2016.1230148.

Page, J., D. Robinson, N. Morel, and J. L. Scartezzini. 2008. "A Generalised Stochastic Model for the Simulation of Occupant Presence." Energy and Buildings 40: 83-98. doi:10.1016/j.enbuild.2007.01.018.

Rezaee, Roya, Jason Brown, Godfried Augenbroe, and Jinsol Kim. 2015. "Assessment of Uncertainty and Confidence in Building Design Exploration." Artificial Intelligence for Engineering Design, Analysis and Manufacturing 29 (04): 429-441. doi:10.1017/S0890060415000426.

Walkenhorst, Oliver, Joachim Luther, Christoph Reinhart, and Jens Timmer. 2002. "Dynamic Annual Daylight Simulations Based on One-Hour and One-Minute Means of Irradiance Data." Solar Energy 72 (5): 385-395. doi:https://doi.org/10.1016/S0038092X(02)00019-1.

\section{Appendix A}

Table A1: Conversion factors site energy to primary energy

\begin{tabular}{|c|c|}
\hline COP & $\begin{array}{c}\text { Conversion } \\
\text { factor }\end{array}$ \\
\hline$\eta_{\text {heating }}$ & 3.00 \\
\hline$\eta_{\text {cooling }}$ & 0.95 \\
\hline$\eta_{\text {electrical }}$ & 0.70 \\
\hline
\end{tabular}

\section{Appendix B}
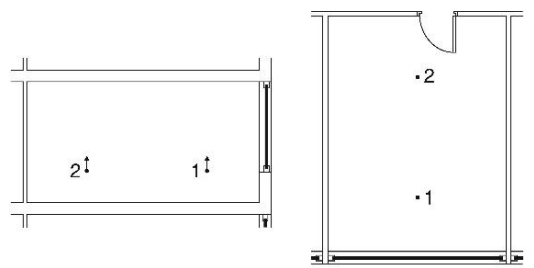

Fig. B1: Sensor points for lighting model

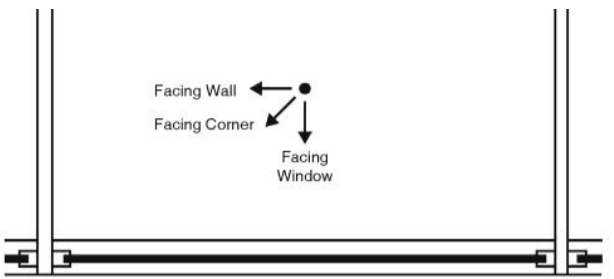

Fig. B2: Sensor point and viewpoints for glare PI 